Dear author,

Please note that changes made in the online proofing system will be added to the article before publication but are not reflected in this PDF.

We also ask that this file not be used for submitting corrections. 
Letters

\title{
The practical clinical relevance of rhinitis classification in children
} Q1 with asthma

\author{
outcomes of the "ControL'Asma" study
}

Asthma and rhinitis may share pathogenic mechanisms, and extensive investigation has been devoted to exploring their reciprocal impact. A recent prospective study investigated the prevalence of rhinitis and its phenotypes, symptom severity, and medication use in 619 children with asthma. ${ }^{1}$ Rhinitis was found to be a common asthma comorbidity (93.5\%) and was refractory to standard rhinitis medications. Perennial allergic rhinitis with seasonal exacerbation caused by poly-allergy was common (34.2\%), mostly severe, and often associated with difficult-to-control asthma. In line with previous evidence, ${ }^{2,3}$ the study concluded that poly-allergy should be considered a significant risk factor for poor control of asthma.

The Italian Society of Paediatric Allergy and Immunology recently established a Study Group ("ControL'Asma”) to evaluate asthma control in children managed in clinical practice. In this context, the group considers rhinitis a comorbidity worthy of investigation. We therefore conducted a study aimed at evaluating the prevalence and impact of rhinitis and its phenotypes on asthma outcomes in a large group of children with asthma.

We enrolled and visited 333 children across 10 Italian paediatric allergy centers. Information was gathered about asthma duration, asthma control levels, and asthma severity grade according to the Global Initiative for Asthma (GINA) guidelines. ${ }^{4}$ Emergency department admissions, absences from school, current use of medications, including inhaled and oral corticosteroids, were also reported, and also body mass index (BMI) assessment. lung function testing, fractinoal exhaled nitric oxide (FeNO) measurement, and children's asthma control test score (c-ACT). Children self-administered the children asthma control test (c-ACT) questionnaire. The Review Ethics Committees approved the study procedure, and written informed consent was obtained from the parents of all children. Clinical data were recorded on an electronic case report form approved for this study.

Demographic and clinical characteristics are described using means with standard deviation for normally distributed continuous data (eg, age), medians with lower and upper quartiles for not normally distributed data (eg, FeNO levels), and absolute frequency

\section{Disclosures: none.}

Funding Sources: none.

"ControL'Asma Study Group" members: Elisa Anastasio, Ilaria Brambilla, Carlo Caffarelli, Loredana Chini, Riccardo Ciprandi, Paolo Del Barba, Valentina De Vittori, Maria Elisa Di Cicco, Luciana Indinnimeo, Ahmad Kantar, Maddalena Leone, Amelia Licari, Viviana Moschese, Roberta Olcese, Diego Peroni, Angela Pistorio, Michela Silvestri, Anna Maria Zicari.

Author's contribution: MAT, MD, GM, and GC designed the study, GC wrote the manuscript. and percentages for categorical data (eg, frequency of male subjects). The normality of distribution was assessed by Shapiro-Wilk $\mathrm{W}$ test. Normally distributed quantitative data were analyzed using analysis of variance (ANOVA) followed by a Sheffè post hoc test, and non-normally distributed quantitative data using a Kruskall-Wallis test followed by Bonferroni's correction. Comparison of frequency distributions was made by means of the $\chi^{2}$ test or Fisher's exact test in case of expected frequencies less than 5 , followed by Bonferroni's correction. Statistical significance was set at $P<.05$, and the analyses were performed using GraphPad Prism software (GraphPad Software Inc, CA).

The most relevant outcomes are reported in Table 1 . We stratified children with asthma, using 2 rhinitis classifications: the traditional method based on the symptom seasonality (such as perennial, seasonal, or mixed) and that proposed by the Allergic Rhinitis and its Impact on Asthma (ARIA) guidelines. ${ }^{2,3}$ Allergic rhinitis (AR) and asthma were diagnosed according to validated criteria defined by guidelines. ${ }^{3,4}$ In addition, we evaluated concordance between the 2 rhinitis classifications.

The findings showed that $88 \%$ of children with asthma had rhinitis as a comorbidity. The ARIA classification presented significant differences among subgroups concerning age, asthma duration, asthma control, asthma severity, oral corticosteroid use in the past year, and dosage of inhaled corticosteroids (ICS; Table 1).

Conventional AR classification shows that some variables were also significantly different, consistently with stratification, such as age, BMI, asthma duration, forced expiratory flow at $25 \%$ to $\mathbf{7 5} \%$ of the pulmonary volume $\left(\mathrm{FEF}_{25-75}\right)$, and c-ACT.

Finally, no concordance was found between the 2 classifications, because there was no overlapping between them without any significant difference.

Rhinitis, particularly allergic rhinitis, is associated with asthma very frequently. The ARIA and conventional classifications are not interchangeable and represent 2 different ways of characterizing $\mathrm{AR}$, as previously reported. ${ }^{5}$ In other words, the information obtained from the classifications is in both cases relevant but may not be consistent.

The ARIA classification was found to be more suitable for characterizing some parameters associated with asthma control and asthma severity. Significant differences were seen among the rhinitis phenotypes in features such as age, asthma duration, use of oral corticosteroids, asthma exacerbations, and ICS dosage. In short, the ARIA classification provides clear indications about the impact of rhinitis phenotyping on asthma severity.

Conventional classification identified parameters that differentiate the rhinitis phenotypes. Significant differences among groups

1081-1206/@ 2019 American College of Allergy, Asthma \& Immunology. Published by Elsevier Inc. All rights reserved. 


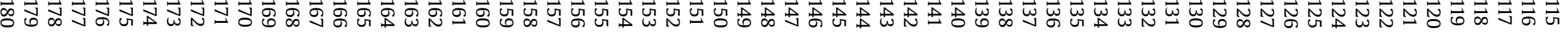

Table 1

Comparisons among Different Asthmatic Groups with or without Allergic Rhinitis, Stratified According to ARIA or Conventional Classification

\begin{tabular}{|c|c|c|c|c|c|c|c|}
\hline \multirow[t]{2}{*}{ Characteristic } & \multicolumn{7}{|l|}{ ARIA classification } \\
\hline & $\begin{array}{l}\text { No rhinitis (NoR) } \\
(\mathrm{n}=39 ; 11.71 \%)\end{array}$ & $\begin{array}{l}\text { Nonallergic } \\
\text { rhinitisAllergic } \\
\text { Rhinitis (NAR) } \\
(\mathrm{n}=12 ; 3.60 \%)\end{array}$ & $\begin{array}{l}\text { Mild intermittent } \\
\text { allergic rhinitis (MIAR) } \\
(\mathrm{n}=100 ; 30.03 \%)\end{array}$ & $\begin{array}{l}\text { Moderate-severe } \\
\text { intermittent allergic } \\
\text { rhinitis (MSIAR) } \\
(\mathrm{n}=23 ; 6.91 \%)\end{array}$ & $\begin{array}{l}\text { Mild persistent } \\
\text { allergic rhinitis } \\
\text { (MPAR) } \\
(\mathrm{n}=142 ; 42.64 \%)\end{array}$ & $\begin{array}{l}\text { Moderate-severe } \\
\text { persistent allergic } \\
\text { rhinitis (MSPAR) } \\
(\mathrm{n}=17 ; 5.11 \%)\end{array}$ & $P$ value \\
\hline Age (yrs, mean [SD]) & $9.63(2.87)$ & $9.74(2.59)$ & $11.89(3.09)$ & $11.87(3.42)$ & $11.63(2.88)$ & $11.32(2.65)$ & 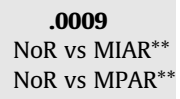 \\
\hline \multicolumn{8}{|l|}{$\operatorname{Sex}(\mathrm{N}[\%])$} \\
\hline Male & \multirow{2}{*}{$\begin{array}{l}27(69.23 \%) \\
12(30.77 \%)\end{array}$} & $10(83.33 \%)$ & $74(74 \%)$ & $14(60.87 \%)$ & $101(71.13 \%)$ & $10(58.82 \%)$ & .59 \\
\hline Female & & $2(16.67 \%)$ & $26(26 \%)$ & $9(39.13 \%)$ & 41 (28.87\%) & $7(41.18 \%)$ & \\
\hline BMI $\left(\mathrm{kg} / \mathrm{m}^{2}\right.$, mean $\left.[\mathrm{SD}]\right)$ & \multirow{2}{*}{$\begin{aligned} 18.56(4.56) \\
19.5(9.3-40)\end{aligned}$} & $18.66(2.19)$ & $20.36(4.10)$ & $20.38(4.10)$ & $19.92(3.92)$ & $19.45(3.97)$ & .21 \\
\hline FeNO (ppb, median [LQ-UQ]) & & $16(8.5-23.5)$ & $21.00(12.50-40.00)$ & $30.00(14.00-36.50)$ & $24.00(13.00-45.00)$ & $31.00(10.50-57.50)$ & .64 \\
\hline Asthma duration (yrs, mean [SD]) & $4.49(3.10)$ & $5.73(2.49)$ & $5.825(3.467)$ & $7.105(3.430)$ & $5.442(3.161)$ & $7.462(5.487)$ & .037 \\
\hline $\mathrm{FEV}_{1}(\%$ pred, mean $[\mathrm{SD}])$ & $93.18(16.4)$ & $94.52(16.02)$ & $94.93(14.90)$ & $101.1(13.87)$ & $97.70(13.83)$ & $95.00(18.42)$ & .30 \\
\hline FVC (\% pred, mean [SD]) & $99.4(16.6)$ & $96.8(13.86)$ & $99.54(14.80)$ & $99.84(13.43)$ & $99.53(12.26)$ & $98.88(16.37)$ & .99 \\
\hline $\mathrm{FEF}_{25-75}$ (\% pred, mean [SD]) & $80.52(20.29)$ & $80.64(23.71)$ & $82.80(24.59)$ & $94.04(23.57)$ & $87.66(24.42)$ & $86.80(22.97)$ & .28 \\
\hline $\mathrm{FVC}_{\mathrm{FEV}}{ }_{1}(\%$ pred, mean [SD]) & \multirow{2}{*}{$19(11.5-24)$} & $99.83(7.334)$ & $95.40(12.69)$ & $97.36(22.63)$ & $98.49(8.699)$ & $97.00(13.51)$ & .50 \\
\hline c-ACT (score, median [LQ-UQ]) & & $19(8-21)$ & $21.00(15.75-24.00)$ & $20.00(18.00-23.00)$ & $20.00(12.00-24.00)$ & $18.00(8.50-22.00)$ & .08 \\
\hline \multicolumn{8}{|l|}{ Asthma control (GINA)(N [\%]) } \\
\hline Well-controlled & $21(53.85 \%)$ & $3(25 \%)$ & $59(59 \%)$ & $7(30.43 \%)$ & $86(60.56 \%)$ & $8(47.06 \%)$ & .035 \\
\hline Poorly controlled & \multirow{2}{*}{$\begin{array}{l}14(35.9 \%) \\
4(10.26 \%)\end{array}$} & $8(66.67 \%)$ & $34(34 \%)$ & $11(47.83 \%)$ & $38(26.76 \%)$ & $5(29.41 \%)$ & \\
\hline Not controlled & & $1(8.33 \%)$ & $7(7 \%)$ & $5(21.74 \%)$ & $18(12.68 \%)$ & $4(23.53 \%)$ & \\
\hline \multicolumn{8}{|l|}{ Asthma severity (GINA) (N [\%]) } \\
\hline Intermittent & $19(48.72 \%)$ & $7(58.33 \%)$ & $62(62 \%)$ & $7(30.43 \%)$ & $48(33.8 \%)$ & 7 (41.18\%) & $<.0001$ \\
\hline Mild persistent & \multirow{2}{*}{$\begin{aligned} 14 & (35.9 \%) \\
5 & (12.82 \%)\end{aligned}$} & $1(8.33 \%)$ & $26(26 \%)$ & $9(39.13 \%)$ & 69 (48.59\%) & $3(17.65 \%)$ & \\
\hline Moderate persistent & & $4(33.33 \%)$ & $10(10 \%)$ & $5(21.74 \%)$ & $25(17.61 \%)$ & $7(41.18 \%)$ & \\
\hline Severe persistent & $1(2.56 \%)$ & $0(0 \%)$ & $2(2 \%)$ & $2(8.7 \%)$ & $0(0 \%)$ & $0(0 \%)$ & \\
\hline $\begin{array}{l}\text { Absence from school (only when } \\
\text { reported)(N, median [LQ-UQ]) }\end{array}$ & $2.5(1.5-10.5)$ & $3(-)$ & $4.00(1.50-6.50)$ & $8.50(5.00-14.00)$ & $4.00(2.00-6.00)$ & $4.00(1.50-17.50)$ & .46 \\
\hline ED admission (at least one) ( $\mathrm{N}[\%])$ & $4(10.53 \%)$ & $0(0 \%)$ & $5(5.1 \%)$ & $0(0 \%)$ & $9(6.43 \%)$ & $2(12.5 \%)$ & .43 \\
\hline $\begin{array}{l}\text { Oral CS (only in positive cases) (N, } \\
\text { median [LQ-UQ]) }\end{array}$ & $1(1-1)$ & $2(-)$ & $1.00(1.00-2.00)$ & $3.00(1.00-4.00)$ & $1.00(1.00-4.00)$ & $1.00(1.00-1.00)$ & .0491 \\
\hline \multicolumn{8}{|l|}{ ICS dose $(\mathrm{N}[\%])$} \\
\hline No ICS & \multirow{2}{*}{$\begin{array}{r}7(18.42 \%) \\
20(52.63 \%)\end{array}$} & $0(0 \%)$ & $30(30.93 \%)$ & $6(26.09 \%)$ & $40(28.57 \%)$ & $4(25 \%)$ & .0052 \\
\hline Low-dose & & $4(33.33 \%)$ & $34(35.05 \%)$ & $7(30.43 \%)$ & $66(47.14 \%)$ & $8(50 \%)$ & \\
\hline Medium-dose & $11(28.95 \%)$ & $8(66.67 \%)$ & 30 (30.93\%) & $7(30.43 \%)$ & $33(23.57 \%)$ & $4(25 \%)$ & \\
\hline High-dose & $0(0 \%)$ & $0(0 \%)$ & $3(3.09 \%)$ & $3(13.04 \%)$ & $1(0.71 \%)$ & $0(0 \%)$ & \\
\hline Characteristic & & ional AR classificat & & & & & $P$ value \\
\hline & & $\begin{array}{l}\text { tis (NoR) } \\
11.96 \%)\end{array}$ & $\begin{array}{l}\text { Non allergic rhinitis } \\
\text { (NAR) }(\mathrm{n}=12 ; 3.68 \%)\end{array}$ & $\begin{array}{l}\text { Mixed allergic } \\
\text { rhinitis (MAR) } \\
(\mathrm{n}=190 ; 58.28 \%)\end{array}$ & $\begin{array}{l}\text { Perennial allergic } \\
\text { rhinitis (PAR) } \\
(\mathrm{n}=66 ; 20.25 \%)\end{array}$ & $\begin{array}{l}\text { Seasonal allergic } \\
\text { rhinitis (SAR) } \\
(\mathrm{n}=19 ; 5.83 \%)\end{array}$ & \\
\hline Age (yrs, mean [SD]) & & 87) & $9.74(2.59)$ & $12.17(2.82)$ & $10.79(2.99)$ & $10.59(3.40)$ & $\begin{array}{l}<.0001 \\
\text { NoR vs MAR } \\
\text { MAR vs PAR* }\end{array}$ \\
\hline $\operatorname{Sex}(N[\%])$ & & & & & & & \\
\hline Male & & $9.23 \%)$ & $10(83.33 \%)$ & 135 (71.05\%) & 47 (71.21\%) & $14(73.68 \%)$ & .91 \\
\hline Female & & $0.77 \%)$ & $2(16.67 \%)$ & 55 (28.95\%) & $19(28.79 \%)$ & $5(26.32 \%)$ & \\
\hline BMI $\left(\mathrm{kg} / \mathrm{m}^{2}\right.$, mean $\left.[\mathrm{SD}]\right)$ & & & $18.66(2.19)$ & $20.27(4.06)$ & $19.91(3.69)$ & $19.13(3.84)$ & $\begin{array}{l}.0235 \\
\text { NoR vs MAR* }\end{array}$ \\
\hline FeNO (ppb, median [LQ-UQ]) & & $3-40)$ & $16(8.5-23.5)$ & $24(14.5-40)$ & $24.8(8-56)$ & $14(12-33)$ & .31 \\
\hline Asthma duration (yrs, mean [SD]) & & 10) & $5.73(2.49)$ & $6.32(3.37)$ & $4.69(3.74)$ & $5.6(3.27)$ & $\begin{array}{l}.0018 \\
\text { NoR vs MAR* } \\
\text { MAR vs PAR** }\end{array}$ \\
\hline
\end{tabular}

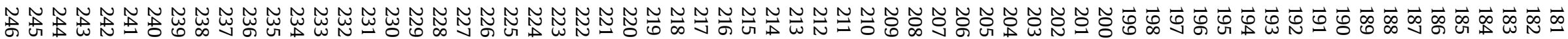




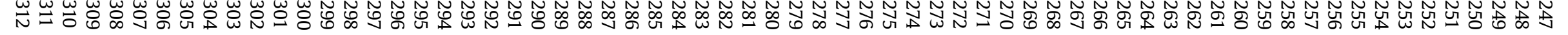

\begin{tabular}{|c|c|c|c|c|c|c|}
\hline $\mathrm{FEV}_{1}(\%$ pred, mean $[S D])$ & $93.18(16.4)$ & $94.52(16.02)$ & $98.07(13.7)$ & $93.22(16.04)$ & $100.3(14.61)$ & .09 \\
\hline FVC (\% pred, mean [SD]) & $99.4(16.6)$ & $96.8(13.86)$ & $100.4(12.82)$ & $98.58(14.3)$ & $98.21(15.36)$ & .82 \\
\hline $\mathrm{FEF}_{25-75}(\%$ pred, mean $[\mathrm{SD}])$ & $80.52(20.29)$ & $80.64(23.71)$ & $87.19(24.23)$ & $80.67(22.39)$ & $101.7(21.89)$ & $\begin{array}{l}.009 \\
\text { NoR vs SAR* } \\
\text { PAR vs SAR* }\end{array}$ \\
\hline FVC/FEV 1 (\% pred, mean [SD]) & $96.25(12.49)$ & $99.83(7.334)$ & $97.42(12.73)$ & $94.88(11.13)$ & $102.6(9.346)$ & .15 \\
\hline c-ACT (score, median [LQ-UQ]) & $19(11.5-24)$ & $19(8-21)$ & $20(14.5-24)$ & $20(12-23)$ & $23.5(15.5-24.5)$ & $\begin{array}{l}.0436 \\
\text { NAR vs SAR* }\end{array}$ \\
\hline \multicolumn{7}{|l|}{ Asthma control (GINA) (N [\%]) } \\
\hline Well-controlled & $21(53.85 \%)$ & $3(25 \%)$ & $109(57.37 \%)$ & 35 (53.03\%) & $14(73.68 \%)$ & .22 \\
\hline Poorly controlled & $14(35.9 \%)$ & $8(66.67 \%)$ & $59(31.05 \%)$ & $22(33.33 \%)$ & $5(26.32 \%)$ & \\
\hline Not controlled & $4(10.26 \%)$ & $1(8.33 \%)$ & $22(11.58 \%)$ & $9(13.64 \%)$ & $0(0 \%)$ & \\
\hline \multicolumn{7}{|l|}{ Asthma severity (GINA) (N [\%]) } \\
\hline Intermittent & 19 (48.72\%) & $7(58.33 \%)$ & $83(43.68 \%)$ & 27 (40.91\%) & $12(63.16 \%)$ & .34 \\
\hline Mild persistent & $14(35.9 \%)$ & $1(8.33 \%)$ & $72(37.89 \%)$ & $25(37.88 \%)$ & $6(31.58 \%)$ & \\
\hline Moderate persistent & $5(12.82 \%)$ & $4(33.33 \%)$ & $33(17.37 \%)$ & $13(19.7 \%)$ & $0(0 \%)$ & \\
\hline Severe persistent & $1(2.56 \%)$ & $0(0 \%)$ & $2(1.05 \%)$ & $1(1.52 \%)$ & $1(5.26 \%)$ & \\
\hline $\begin{array}{l}\text { Absence from school (only in positive cases) ( } \mathrm{N} \text {, } \\
\text { median [LQ-UQ]) }\end{array}$ & $2.5(1.5-10.5)$ & $3(-)$ & $4(2-5)$ & $8(1.5-17.5)$ & $5(-)$ & .24 \\
\hline $\begin{array}{l}\text { ED admission (only in positive cases) (N, } \\
\text { median [LQ-UQ]) }\end{array}$ & $1.5(-)$ & $0(-)$ & $1(1-1)$ & $1(-)$ & 0 & .12 \\
\hline $\begin{array}{l}\text { Oral CS (only in positive cases) ( } \mathrm{N} \text {, median [LQ- } \\
\text { UQ]) }\end{array}$ & $1(1-1)$ & $2(-)$ & $1(1-2.5)$ & $1(1-4)$ & $1.5(-)$ & .34 \\
\hline \multicolumn{7}{|l|}{ ICS dose (N [\%]) } \\
\hline No ICS & $7(18.42 \%)$ & $0(0 \%)$ & $55(29.1 \%)$ & $18(28.13 \%)$ & $7(38.89 \%)$ & .10 \\
\hline Low dose & $20(52.63 \%)$ & $4(33.33 \%)$ & $79(41.8 \%)$ & $27(42.19 \%)$ & $7(38.89 \%)$ & \\
\hline Medium dose & $11(28.95 \%)$ & $8(66.67 \%)$ & $52(27.51 \%)$ & $16(25 \%)$ & $3(16.67 \%)$ & \\
\hline High dose & $0(0 \%)$ & $0(0 \%)$ & $3(1.59 \%)$ & $3(4.69 \%)$ & $1(5.56 \%)$ & \\
\hline \multicolumn{7}{|l|}{ ARIA classification (N [\%]) } \\
\hline MIAR & - & - & $65(34.21 \%)$ & $26(39.39 \%)$ & $7(36.84 \%)$ & .53 \\
\hline MSIAR & - & - & 17 (8.95\%) & $4(6.06 \%)$ & $2(10.53 \%)$ & \\
\hline MPAR & - & - & $98(51.58 \%)$ & 29 (43.94\%) & $10(52.63 \%)$ & \\
\hline MSPAR & - & - & $10(5.26 \%)$ & $7(10.61 \%)$ & $0(0 \%)$ & \\
\hline
\end{tabular}

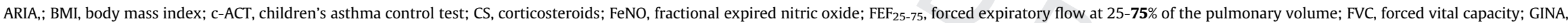
Global Initiative for Asthma; ICS, inhaled corticosteroids; LQ lower quadrant: SD, standard deviation; UQ upper quadrant. 
were found for age, BMI, asthma duration, c-ACT score, and FEF $25-75$ value, the last of which may be considered an early predictor of bronchial airflow limitation. ${ }^{6}$ These results show that children without rhinitis (NoR) were younger, had lower BMI and $\mathrm{FEF}_{25-75}$, and had shorter asthma duration than did the other subjects. More interestingly, children with mixed allergic rhinitis (MAR) had longer asthma duration (consistent with poly-allergy), whereas children with seasonal allergic rhinitis (SAR) had higher $\mathrm{FEF}_{25-75}$ than children with perennial allergic rhinitis (PAR) (consistent with shorter allergen exposure). Therefore, the conventional classification of rhinitis may be useful for providing indications about the impact of rhinitis phenotypes on clinical and functional variables in these children with asthma.

Comparing the findings obtained from the 2 rhinitis classifications, consistent results were obtained only for age and asthma duration: younger children and those with shorter asthma duration more frequently did not present rhinitis comorbidity. The ARIA classification better identified significant differences among rhinitis phenotypes concerning asthma control and severity scoring. On the contrary, conventional classification better identified notable differences among rhinitis phenotypes regarding the subjective perception of asthma control (c-ACT) and the functional parameter $\mathrm{FEF}_{25-75}$.

The results of this study suggest that the 2 rhinitis classifications provide different information about children with asthma, and that both information sets can generate clinically relevant data that may be useful in asthma management. In clinical practice, using both rhinitis classifications would seem to be more helpful than considering only 1 . In particular, rhinitis is well known to have a profound impact on asthma, mainly concerning the upper airways treatment. Therefore, phenotyping rhinitis in children with asthma could drive therapeutic strategies directed toward nasal inflammation dampening. The current study suggests that poly-allergy and severe rhinitis are factors associated with poorly controlled and severe asthma.

The main limitation of this study is its cross-sectional design, but follow-up is ongoing.
In conclusion, rhinitis is frequently associated with asthma in children; rhinitis phenotyping should be performed using both conventional and ARIA classification; and rhinitis phenotyping allows clinicians to diagnose and treat the asthma properly.

Maria Angela Tosca, $\mathrm{PhD}$ Marzia Duse, MD

Gianluigi Marseglia, MD

Giorgio Ciprandi, MD ${ }^{\S}$ “ControL’Asma” Study Group *Pediatric Allergy Center Istituto Giannina Gaslini Genoa, Italy

${ }^{\dagger}$ Pediatrics Department Umberto I Hospital Roma Sapienza University Roma, Italy

${ }_{\ddagger}^{\ddagger}$ Pediatrics Clinic, Pediatrics Department, Policlinico San Matteo, University of Pavia, Pavia, Italy ${ }^{\S}$ Allergy Clinic Casa di Cura Villa Montallegro Genoa, Italy gio.cip@libero.it

\section{References}

1. Togias A, Gergen PJ, Hu JW, et al. Thinitis in children and adolescents with asthma: ubiquitous, difficult to control, and associated with asthma outcomes. J Allergy Clin Immunol. 2018. https://doi.org/10.1016/j.jaci.2018.06.043.

2. Ciprandi G, Cirillo I, Vizzaccaro A, Tosca MA, Pallestrini E. Seasonal and perennial allergic rhinitis: is this classification adherent to real life? A population based study. Allergy. 2005;60:882-887.

3. Brożek JL, Bousquet J, Agache I, et al. Allergic Rhinitis and its Impact on Asthma (ARIA) guidelines: 2016 revision. J Allergy Clin Immunol. 2017;140(4):950-958.

4. Global Initiative for Asthma. GINA guidelines. Global strategy for Asthma Management and Prevention. 2018. Available at: htpp://www.ginasthma.org/. Accessed June 2019.

5. Bauchau V, Durham SR. Epidemiological characterization of the intermittent and persistent types of allergic rhinitis. Allergy. 2005;60(3):350-353.

6. Ciprandi G, Cirillo I. The lower airway pathology of rhinitis: update review. J Allergy Clin Immunol. 2006;118:1105-1109. 